\section{Hemodiafiltración intermitente en línea como terapia de reemplazo renal en paciente crítico: Experiencia en un centro de diálisis de agudo}

\author{
SILVIA BARRIOS ARAYA ${ }^{1, a}$, MARÍA ELISA ESPINOZA COYA2,a, \\ KELLY ÑUÑEZ GÓMEZ2,a, RODRIGO SEPÚLVEDA PALAMARA ${ }^{3, \mathrm{~b}}$, \\ YERKO MOLINA MUÑOZ ${ }^{1, c}$
}

\section{Experience with inline intermittent hemodiafiltration as renal replacement therapy in critically ill patients}

Background: In critical patients with acute renal failure, intermittent diffusive renal replacement techniques cause hemodynamic problems due to their high depurative efficiency. This situation is avoided using continuous low efficiency therapies, which are expensive, prevent patient mobilization and add hemorrhagic risk due to systemic anticoagulation. Intermittent and prolonged hemodiafiltration (HDF) has the depurative benefits of diffusion, plus the positive attributes of convection in a less expensive therapy. Aim: To report our experience with intermittent and prolonged on-line HDF in critically ill patients. Patients and Methods: During 2016, HDF therapies performed on critical patients with indication of renal replacement therapy were characterized. The hemodynamic profile was evaluated (doses of noradrenaline, blood pressure, heart rate and perfusion parameters). Results: Fifty-one therapies were performed in 25 critical patients, aged $58 \pm 11$ years (28\% women), with an APACHE II score of 22.1 \pm 10 . The average time of the therapies was 4.15 hours (range 3-8 hours), the replacement volume was $75 \pm 18 \mathrm{~mL} / \mathrm{kg} / \mathrm{h}$ and ultrafiltration rate was $226 \pm 207$ $\mathrm{mL} / \mathrm{h}$. The mean initial, maximum and final noradrenaline doses were $0.07 \pm$ $0.1,0.13 \pm 0.18$ and $0.09 \pm 0.16 \mu \mathrm{g} / \mathrm{kg} / \mathrm{min}$ respectively. No differences between patients with low, medium and high doses of noradrenaline or dose increases during therapy, were observed. The greatest decrease in mean arterial pressure was $15.3 \%$ and the maximum increase in heart rate was $12.8 \%$. Anticoagulation was not required in $88 \%$ of therapies. Conclusions: High-volume intermittent or prolonged HDF is an effective therapy in critical patients, with good hemodynamic tolerability, lower costs and avoidance of systemic anticoagulation risks.

(Rev Med Chile 2019; 147: 409-415)

Key words: Acute kidney injury; Critically Ill; Hemodiafiltration.

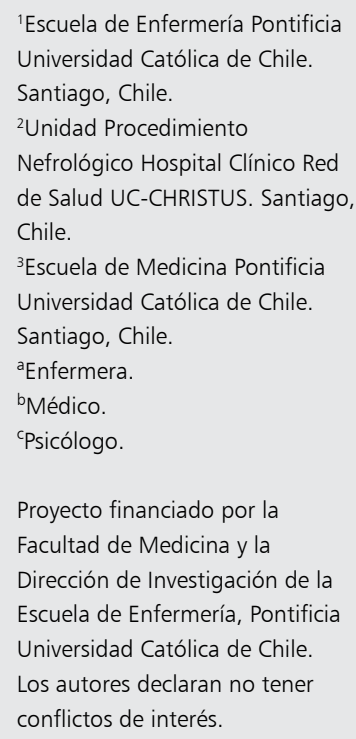

Proyecto financiado por la Facultad de Medicina y la Dirección de Investigación de la Escuela de Enfermería, Pontificia Universidad Católica de Chile. Los autores declaran no tener conflictos de interés.

Recibido el 3 de abril de 2018, aceptado el 1 de abril de 2019 .

Correspondencia a: Kelly Núñez Gómez Lira 40. Unidad Procedimiento Nefrológico Hospital Clínico Red de Salud UC-CHRISTUS-Santiago de Chile. Santiago, Chile.

kelly.nunez.gomez@gmail.com

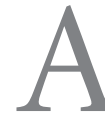

ctualmente se dispone de diferentes terapias de reemplazo renal (TRR), desde técnicas difusivas como hemodiálisis (HD), técnicas convectivas como hemofiltración (HF) y una combinación de éstas: hemodiafiltración (HDF).
Las técnicas difusivas emplean el principio de difusión para traspaso de moléculas entre dos compartimientos (sangre y compartimiento de diálisis) separados por una membrana semipermeable. Las terapias convectivas producen un 
ultrafiltrado de plasma desde la sangre (efluente), el que luego será repuesto con un líquido que posee concentraciones óptimas de los principales solutos del plasma.

Las técnicas difusivas permiten una depuración más eficiente que las convectivas porque extraen rápidamente solutos (drogas, compuestos nitrogenados, potasio, ácidos, etc.). Esto genera disminución de la osmolaridad plasmática y desplazamiento de líquido al intracelular. Además, inhibe la secreción de vasopresina y por mecanismos desconocidos disminución de la actividad simpática ${ }^{1,2}$. Así, la depuración repercute negativamente en la hemodinamia al contraer el intravascular (por escape de líquido hacia el intracelular) y disminución del tono vasomotor. Todo esto, sin considerar el balance hídrico (BH) negativo extraído directamente desde el intravascular.

Mientras más eficiente la depuración, mayor compromiso hemodinámico, por lo cual las terapias continuas $(>24 \mathrm{~h}$ ) de muy baja eficiencia son la modalidad de elección en pacientes críticos con compromiso hemodinámico. En terapias convectivas, el líquido de sustitución induce hipotermia (favoreciendo vasoconstricción y redistribución de flujo); mejorando el estado hemodinámico.

La literatura sobre TRR en paciente crítico reporta que las terapias más utilizadas son $\mathrm{HF}, \mathrm{HD}$ o HDF venovenosa continua (CVV). En europa se utiliza principalmente CVVHF, en Australia y Nueva Zelanda CVVHDF, mientras que en Norteamérica se agrega la $\mathrm{CVVHD}^{3-5}$, Un estudio realizado en 54 Unidades de Cuidado Intensivo (UCI) reporta mayor uso de CVVHF (53\%) que CVVHDF $(34 \%)^{6}$.

Los problemas de las terapias continuas son la anticoagulación sistémica con riesgo hemorrágico, inmovilización del paciente dificultando realizar procedimientos o exámenes imagenológicos y altos costos por necesidad de recurso humano especializado. Las terapias intermitentes $(<6 \mathrm{~h})$ y prolongadas $(>6 \mathrm{~h})$ no presentan estas dificultades. Revisiones sistemáticas y estudios prospectivos no han encontrado diferencias en morbimortalidad respecto a terapias continuas versus intermitentes/prolongadas ${ }^{7-9}$.

La HDF intermitente/prolongada tiene los beneficios de la HF, agrega la eficiencia depurativa de la HD y evita las dificultades de las terapias continuas. En la HDF en línea el líquido de sustitución se produce simultáneamente a partir del líquido de diálisis, que previamente pasa por dos filtros en serie, cuyo objetivo es impedir el paso de potenciales toxinas y contaminantes dada su capacidad de absorción; esta característica garantiza una solución ultra pura que puede ser infundida directamente al torrente sanguíneo del paciente ${ }^{10}$. La sustitución pre-dilución permite utilizar flujo de sangre $(\mathrm{Qb})$ bajo con menor riesgo de coagulación, la convección del plasma mediante filtros de alto flujo permite la remoción de citoquinas que tienen propiedades inflamatorias y vasodilatadoras. La menor temperatura del líquido de sustitución y la facilidad para programar una mayor concentración de sodio en el líquido de diálisis y sustitución $(>140 \mathrm{mEq} / \mathrm{L})$ permiten una mejor tolerancia hemodinámica ${ }^{11}$. Otro factor beneficioso es la disminución de los costos en salud. Las terapias continuas requieren varias horas a la semana personal entrenado, lo que se evita con terapias prolongadas e intermitentes. Además, la producción "en línea" de líquido de diálisis y sustitución, también contribuyen a disminuir los $\operatorname{costos}^{10,12}$.

Pese a atributos favorables, es una terapia destinada a enfermos renales crónicos, y no, a insuficiencia renal aguda (IRA); por lo que la literatura sobre su uso en paciente crítico es escasa.

Por las características mencionadas, la tolerabilidad hemodinámica de una HDF intermitente o prolongada, debiera ser mejor que una HD intermitente; y comparable, si no equivalente, a una terapia continua.

A continuación, presentamos nuestra experiencia sobre el uso de HDF intermitente y prolongada en pacientes críticos, con énfasis en la tolerabilidad hemodinámica a la terapia.

\section{Metodología}

Se caracterizaron en forma prospectiva pacientes críticos que requirieron TRR con HDF en un hospital docente de la Región Metropolitana durante el año 2016. Se incluyeron pacientes críticos (ingresados a UCI o Intermedio) con diagnóstico de IRA o enfermedad renal crónica terminal (ERCT) en HD que requirieran TRR. Los pacientes además portaban algún otro factor para considerar HDF; como estados hipercatabólicos o necesidad de mayor depuración. La indicación de HDF, filtro, tiempo, flujo de diálisis (Qd) y Qb 
quedaba a juicio del nefrólogo tratante. En los individuos seleccionados se aplicó un consentimiento informado (paciente o tutor legal).

Se excluyeron pacientes críticos menores de 18 años, embarazadas, aquellos con serología viral positiva (para hepatitis $\mathrm{B}, \mathrm{C}$ y VIH), y pacientes con shock muy grave (noradrenalina [NAD] $>0,3-0,4 \mu \mathrm{g} / \mathrm{kg} / \mathrm{min}$ ) porque en ellos se realizaron terapias convectivas (HF prolongada, HF de alto volumen o CVVHF).

Se obtuvieron los siguientes datos de los pacientes: antecedentes demográficos, antropométricos, diagnóstico de ingreso, gravedad según score APACHE II, condición de egreso y recuperabilidad de función renal.

Durante cada terapia de HDF se realizó monitorización continua con registro cada 15 minutos de presión arterial (PA) sistólica, diastólica y media (PAM), frecuencia cardiaca (FC), temperatura, requerimiento y dosis de drogas vasoactivas, parámetros de la TRR (tiempo, Qb, Qd, sustitución, filtro, $\mathrm{BH}$, composición del líquido de diálisis, temperatura), complicaciones durante la terapia, necesidad de suspenderla o cambio a HF, necesidad y dosis de anticoagulación y coagulación del filtro. Se registraron parámetros perfusionales como lactato y saturación venosa central de oxígeno $\left(\mathrm{SVcO}_{2}\right)$. Se clasificaron las terapias realizadas a los pacientes críticos según el grado de compromiso hemodinámico que presentaban: ausente, leve, intermedio o severo. Esto último definido por los requerimientos de $\mathrm{NAD}$ al ingreso: sin requerimientos, requerimiento de bajas dosis $(\leq 0,1 \mu \mathrm{g} /$ $\mathrm{kg} / \mathrm{min})$, media $(>0,1 \mathrm{y}<0,3 \mu \mathrm{g} / \mathrm{kg} / \mathrm{min})$ y alta $(\geq 0,3 \mu \mathrm{g} / \mathrm{kg} / \mathrm{min})$ respectivamente.

Las HDF fueron realizadas por un equipo profesional capacitado para realizar TRR en paciente crítico, con un equipo Dialog Plus de B. Braun ${ }^{\circledR}$ y filtros de alto flujo (superficie 1,5, 1,8 y $2 \mathrm{~m}^{2}$ ).

Este estudio cumple con la Declaración Internacional de Helsinki y fue aprobado por el comité de ética de la Pontificia Universidad Católica de Chile.

El análisis estadístico se realizó mediante software IBM-SPSS ${ }^{\circledR}$ versión 24 y Microsoft Excel $^{\circledR}$. Variables numéricas se caracterizaron con media, desviaciones estándar y rangos. Variables categóricas se describieron mediante frecuencias. Se realizaron comparaciones de datos categóricos y continuos utilizando prueba T de Student y ANOVA. Para asociación de variables continuas se estimó una correlación lineal de Pearson. Se utilizó un valor p significativo menor de $5 \%$.

\section{Resultados}

Durante el año 2016 hubo 877 TRR en pacientes críticos y en 52 de éstas (5,93\%) se indicó HDF intermitente o prolongada. Se excluyó uno con indicación de HDF prolongada, que posteriormente se conectó a HF por rápido compromiso hemodinámico. La Tabla 1 muestra las características de los pacientes, y la Tabla 2 las medidas de resumen de todas las terapias HDF realizadas.

Se realizaron $51 \mathrm{HDF}$ en 25 pacientes críticos; $28 \%$ eran mujeres con edad promedio 58,12 $( \pm 11,17), 12 \%$ de los pacientes críticos sometidos a HDF presentaban una IRA KDIGO III sobre enfermedad renal crónica (ERC), 24\% tenían previamente una ERCT en HD y $64 \%$ se sometieron

\section{Tabla 1. Características de los pacientes a quienes se realizó HDF $(n=25)$}

\begin{tabular}{|lc|}
\hline Pacientes & \\
\hline Edad & $78,12( \pm 11,17)$ años \\
\hline Sexo Femenino * & $7(28 \%)$ \\
\hline Diagnóstico de Ingreso* & \\
- Sepsis y shock séptico & $10(40 \%)$ \\
- Enfermedad pulmonar & $2(8 \%)$ \\
- Patología hepática & $3(12 \%)$ \\
- Enfermedad cardiovascular & $5(20 \%)$ \\
- Enfermedad hémato-oncológica & $1(4 \%)$ \\
- Traumatismos & $2(8 \%)$ \\
- Enfermedad intestinal & $2(8 \%)$ \\
\hline Patología médica* & $16(64 \%)$ \\
Patología quirúrgica* & $9(36 \%)$ \\
\hline Diagnóstico renal* & \\
- Paciente crítico con ERCT en diálisis & $6(24 \%)$ \\
- Paciente crítico con IRA sobre ERC & $3(12 \%)$ \\
- Paciente crítico con IRA & $16(64 \%)$ \\
\hline APACHE II & $22,12( \pm 10,03)$ \\
\hline Días de hospitalización & $47,5( \pm 31,43)$ \\
\hline Condición de egreso* & \\
- Con hemodiálisis & $8(32 \%)$ \\
- Sin hemodiálisis & $11(44 \%)$ \\
- Fallecido & $5(20 \%)$ \\
- Traslado a otro centro & $1(4 \%)$ \\
\hline Datos presentados con media y desvación & \\
\hline
\end{tabular}

Datos presentados con media y desviación estándar; ${ }^{*} \mathrm{n}$ y porcentaje. 
Tabla 2. Características de las terapias realizadas en pacientes críticos $(\mathbf{n}=\mathbf{5 1})$

\begin{tabular}{|c|c|}
\hline \multicolumn{2}{|l|}{ Terapias en pacientes críticos } \\
\hline $\begin{array}{l}\mathrm{Qd}=300 \mathrm{~mL} / \mathrm{min} * \\
\mathrm{Qd}=500 \mathrm{~mL} / \mathrm{min} *\end{array}$ & $\begin{array}{l}11(26 \%) \\
31(74 \%)\end{array}$ \\
\hline Tiempo & $4: 25( \pm 0: 59)$ \\
\hline Sustitución (mL/kg/min) & $74,64( \pm 17,62)$ \\
\hline Tasa de Ultrafiltración (mL/h) & $226,07( \pm 206,58)$ \\
\hline Efluente (L) & $23,66( \pm 6,52)$ \\
\hline \multicolumn{2}{|c|}{ Hemodinamia de terapias en paciente crítico } \\
\hline $\begin{array}{l}\text { PAM }(\mathrm{mmHg}) \text { : } \\
\text { - Inicial } \\
\text { - Máxima } \\
\text { - Mínima } \\
\text { - Final } \\
\text { - Máxima disminución desde PAM inicial }\end{array}$ & $\begin{array}{l}76,65( \pm 11,36) \\
88,82( \pm 14,83) \\
64,37( \pm 10,75) \\
76,84( \pm 11,87) \\
15,32( \pm 12,97)\end{array}$ \\
\hline $\begin{array}{l}\text { Frecuencia Cardiaca (Ipm): } \\
\text { - Inicial } \\
\text { - Máxima } \\
\text { - Mínima } \\
\text { - Final } \\
\text { - Máximo aumento desde FC inicial }\end{array}$ & $\begin{array}{r}93,47( \pm 18,63) \\
104,73( \pm 20,17) \\
81,14( \pm 18,96) \\
93,1 \quad( \pm 20,14) \\
12,75( \pm 12,45)\end{array}$ \\
\hline $\begin{array}{l}\text { Dosis NAD }(\mu \mathrm{g} / \mathrm{kg} / \mathrm{min}) \text { : } \\
\text { - Dosis NAD inicial } \\
\text { - Dosis NAD máxima } \\
\text { - Dosis NAD final }\end{array}$ & $\begin{array}{l}0,068( \pm 0,1) \\
0,13 \quad( \pm 0,18) \\
0,095( \pm 0,16)\end{array}$ \\
\hline $\begin{array}{l}\text { Lactato previo }(\mathrm{mmol} / \mathrm{L}) \\
\text { Lactato posterior }(\mathrm{mmol} / \mathrm{L})\end{array}$ & $\begin{array}{ll}3,14 & ( \pm 3,98) \\
2,21 & ( \pm 2,02)\end{array}$ \\
\hline $\begin{array}{l}\text { Saturación venosa central previa (\%) } \\
\text { Saturación venosa central posterior (\%) }\end{array}$ & $\begin{array}{l}75,2( \pm 8,27) \\
74,28( \pm 8,29)\end{array}$ \\
\hline
\end{tabular}

Datos presentados con media y desviación estándar; *n y porcentaje.

a HDF por IRA KDIGO III. El score APACHE II promedio fue $22,12( \pm 10,03)$ y $26,94( \pm 8,27)$ en el subgrupo de pacientes con IRA KDIGO III. Días de hospitalización promedio 47,5 $( \pm 31,43)$. De los pacientes con IRA o IRA sobre ERC 10,5\% quedó dependiente de TRR al alta. No hubo fallecimientos entre los pacientes con ERCT ni IRA sobre ERC, sin embargo, en el subgrupo de pacientes con IRA KDIGO III aislada 31,3\% falleció.

En todas las HDF se utilizaron filtros de alto flujo, Qb entre 200-300 mL/min y sustitución $100 \%$ pre-dilución. El tiempo promedio de terapia fue 4:25 con un rango entre 3:00 y 8:00 h. El volumen de sustitución promedio fue $74,6 \mathrm{~mL} / \mathrm{kg} / \mathrm{h}$ $( \pm 17,62), \mathrm{BH}$ negativo con extracción promedio de $1.006 \mathrm{~mL}( \pm 887,2)$ y tasa de ultrafiltración promedio de $226 \mathrm{~mL} / \mathrm{h}( \pm 206,58)$. El efluente promedio fue $23,66 \mathrm{~L}( \pm 6,52)$. En 16 terapias $(38 \%)$ el $\mathrm{BH}$ indicado fue neutro. En el 55\% de las terapias se logró el balance indicado inicialmente, en 31,4\% se sobrepasó por adecuada tolerancia y necesidad de mayor extracción de líquido, y en $13,7 \%$ no se logró por diversos motivos (disfunción de catéter, procedimientos o exámenes imagenológicos y compromiso hemodinámico).

Respecto al comportamiento hemodinámico, la única droga vasoactiva utilizada fue NAD. La dosis promedio de NAD antes de iniciar la HDF fue $0,068 \mu \mathrm{g} / \mathrm{kg} / \mathrm{min}( \pm 0,1)$. La PAM promedio inicial fue $76,65 \mathrm{mmHg}( \pm 11,36)$ y la FC 93,47 $\operatorname{lpm}( \pm 18,6)$. El máximo requerimiento de NAD promedio de las terapias fue $0,128 \mu \mathrm{g} / \mathrm{kg} / \mathrm{min}$ $( \pm 0,18)$, mayor disminución de PAM fue en promedio $15,32 \%( \pm 11,34)$ y máximo aumento de FC promedio 12,75\% ( $\pm 12,45)$. Al finalizar las terapias, la dosis promedio de NAD requerida fue $0,095 \mu \mathrm{g} / \mathrm{kg} / \mathrm{min}( \pm 0,16)$, PAM $76,84 \mathrm{mmHg}$ $( \pm 11,87)$ y FC $93,47 \mathrm{lpm}( \pm 20,14)$. La concentración de lactato previo a la terapia fue $3,14 \mathrm{mmol} / \mathrm{L}$ $( \pm 3,96)$ y $\mathrm{SVcO}_{2} 75,2 \%( \pm 8,27)$.

Se dividieron las terapias en grupos según dosis inicial de NAD: sin requerimientos $(0 \mu \mathrm{g} / \mathrm{kg} / \mathrm{h})$, dosis bajas $(\leq 0,1 \mu \mathrm{g} / \mathrm{kg} / \mathrm{h})$, dosis media $(>0,1 \mathrm{y}$ $<0,3 \mu \mathrm{g} / \mathrm{kg} / \mathrm{h})$ y dosis alta $(>0,3 \mu \mathrm{g} / \mathrm{kg} / \mathrm{h})$. La Tabla 3 caracteriza el comportamiento hemodinámico en las distintas terapias según dosis inicial de NAD. Durante cada terapia, se registró la diferencia de dosis de NAD al inicio y final de la terapia $\left(\triangle \mathrm{NAD}_{\mathrm{f}}\right)$, diferencia de dosis máxima requerida de NAD durante la terapia e inicial $\left(\triangle \mathrm{NAD}_{\max }\right)$, el mayor incremento porcentual en la FC y mayor disminución porcentual de la PAM respecto a los valores iniciales.

Entre los grupos de terapias según dosis inicial de NAD, no hubo diferencia estadística en $\triangle \mathrm{NAD}_{\mathrm{f}}$, ni el máximo aumento porcentual de FC, ni la máxima disminución porcentual de PAM. Sí hubo diferencias significativas en $\triangle \mathrm{NAD}_{\max }$ entre estos grupos. El análisis por comparaciones múltiples mostró que la diferencia significativa estaba entre el grupo sin requerimiento inicial de NAD y los grupos con dosis inicial bajas y altas de NAD ( $\mathrm{p}=0,012$ y $\mathrm{p}=0,006$ respectivamente); no hubo diferencias significativas entre los otros grupos. Al finalizar la terapia; 9,5\% de los casos que inicialmente tenían dosis bajas de NAD, 33,3\% de 
Tabla 3. Comportamiento hemodinámico durante la terapia de HDF según dosis inicial de Noradrenalina

\begin{tabular}{|c|c|c|c|c|c|c|}
\hline & $\begin{array}{c}\text { n de } \\
\text { terapias } \\
(\%)\end{array}$ & $\begin{array}{l}\text { Dosis inicial } \\
\text { ( } \mu \mathrm{g} / \mathrm{kg} / \mathrm{min})\end{array}$ & $\begin{array}{c}\text { ANADmax } \\
(\mu \mathrm{g} / \mathrm{kg} / \mathrm{min})\end{array}$ & $\begin{array}{c}\text { ANADf } \\
(\mu \mathrm{g} / \mathrm{kg} / \mathrm{min})\end{array}$ & $\begin{array}{c}\downarrow \text { PAMmax } \\
\text { (mmHg) }\end{array}$ & $\begin{array}{c}\text { 个FCmax } \\
\text { (Ipm) }\end{array}$ \\
\hline Sin requerimientos & $20(39,2 \%)$ & $( \pm 0)$ & $0,015( \pm 0,06)$ & $( \pm 0)$ & $11,61 \%( \pm 13,08)$ & $7,8 \% \quad( \pm 9,5)$ \\
\hline Dosis bajas & $21(41,2 \%)$ & $0,049( \pm 0,03)$ & $0,057( \pm 0,07)$ & $0,029( \pm 0,04)$ & $14,87 \%( \pm 10,92)$ & $14,1 \% \quad( \pm 12,76)$ \\
\hline Dosis medias & $6(11,8 \%)$ & $0,18 \quad( \pm 0,06)$ & $0,1 * \quad( \pm 0,15)$ & $0,035( \pm 0,11)$ & $24,11 \%( \pm 14,57)$ & $22,53 \%( \pm 11,6)$ \\
\hline Dosis altas & $4(7,8 \%)$ & $0,34 \quad( \pm 0,03)$ & $0,24^{*}( \pm 0,31)$ & $0,14 \quad( \pm 0,38)$ & $23,08 \%( \pm 15,63)$ & $15,8 \% \quad( \pm 18)$ \\
\hline
\end{tabular}

${ }^{*} p<0,05$ en comparaciones múltiples con grupo sin requerimientos de NAD.

aquellos con dosis medias y $50 \%$ de los con dosis altas, disminuyeron sus requerimientos de NAD.

Los valores de $\triangle \mathrm{NAD}_{\mathrm{f}} \mathrm{y} \triangle \mathrm{NAD}$ max reflejan necesidad de mayor dosis de NAD respecto a un basal, y así, indirectamente, mayor compromiso hemodinámico durante la terapia. No hubo correlación lineal significativa entre $\triangle \mathrm{NAD}_{\mathrm{f}} \mathrm{y}$ : APACHE II, edad, lactato, sustitución, $\mathrm{BH}$, aumento máximo porcentual de FC durante la terapia. Se encontró una modesta correlación lineal significativa entre $\triangle \mathrm{NAD}_{\mathrm{f}} \mathrm{y}: \mathrm{SVcO}_{2}$, efluente y disminución máxima porcentual de PAM durante la terapia $(\mathrm{r}=0,42$; $r=-0,44 ; r=0,4$ respectivamente). No hubo correlación lineal significativa entre $\triangle \mathrm{NAD}_{\text {max }} \mathrm{y}$ : APACHE II, edad, lactato, $\mathrm{SVcO}_{2}$, sustitución, $\mathrm{BH}$, efluente, aumento máximo porcentual de FC durante la terapia. Hubo correlación lineal significativa con la máxima disminución porcentual de PAM $(r=0,55)$. No se encontró asociación entre Qd y $\triangle \mathrm{NAD}_{\mathrm{f}}$ ni $\triangle \mathrm{NAD}_{\max }$. Tampoco hubo asociación entre mayor necesidad de dosis de NAD $\left(\triangle \mathrm{NAD}_{\mathrm{f}} \mathrm{y} \Delta \mathrm{NAD}_{\max }\right) \mathrm{y}$ área de superficie del filtro.

Respecto a la anticoagulación, en 6 terapias $(11,76 \%)$ se utilizó heparina no fraccionada en dosis bajas ( 1000 UI en bolo inicial y de mantención). No hubo coagulación de circuitos ni filtros en ninguna terapia y sólo en una terapia $(1,96 \%)$ no se cumplió con el tiempo indicado por disfunción del acceso vascular.

\section{Discusión}

Los factores que influyen en el pronóstico de un paciente crítico son extremadamente diversos y es imposible atribuir sólo a una medida de soporte toda la responsabilidad en el pronóstico. En este sentido, al analizar TRR pareciera apropiado evaluar las características del procedimiento, y no su impacto en la sobrevida o pronóstico, porque esas conclusiones podrían estar sesgadas.

Nuestro estudio comprende un solo centro, sería imprudente realizar un diseño analítico comparativo. La única forma de equilibrar entre grupos todos los factores confundentes, sería realizar un estudio randomizado, multicéntrico y con un mayor número de individuos.

Los reportes de HDF en paciente crítico son muy heterogéneos ${ }^{13-15}$. Nuestros pacientes son similares a los de otras publicaciones; donde la mayoría son hombres con edad promedio de 60 años $^{14,16}$. En el grupo de pacientes críticos con IRA KDIGO III, su gravedad (APACHE II: 26,94) fue similar a lo descrito en la literatura ${ }^{13}$. La mortalidad en este mismo grupo fue $31,3 \%$; mientras que lo reportado en la literatura varía desde $31,6 \%$ hasta $>50 \%{ }^{13,15}$.

La dosis de sustitución utilizada en nuestras terapias de HDF fue de alto volumen (promedio 74,6 $\mathrm{mL} / \mathrm{kg} / \mathrm{h}$ ). Otros autores describen desde $35 \mathrm{~mL} /$ $\mathrm{kg} / \mathrm{h}^{13}$ hasta dosis extremadamente altas: $>120 \mathrm{ml} /$ $\mathrm{kg} / \mathrm{h}$, e incluso, $>200 \mathrm{~mL} / \mathrm{kg} / \mathrm{h}^{15,16}$. Una ventaja de las terapias intermitentes de alto volumen, es que permiten efluentes similares a terapias continuas. Por ejemplo, con $75 \mathrm{~mL} / \mathrm{kg} / \mathrm{h}$ durante $7 \mathrm{~h}$, se logra el mismo efluente que una CVVHF estándar de 22 $\mathrm{ml} / \mathrm{kg} / \mathrm{h}$. Si a esto se agrega el componente difusivo de la HDF, la depuración será mucho mayor. Un inconveniente es la elevada fracción de filtración, sin embargo, como la sustitución es pre-dilución y la terapia intermitente, no tuvimos coagulación del filtro. Por otro lado, según nuestros resultados, la superficie del filtro no incide en la tolerancia hemodinámica, por lo cual es planteable utilizar 
filtros grandes para evitar aumentos de presión en el circuito y riesgo de coagulación.

La literatura reporta un amplio uso de anticoagulación en $\mathrm{HDF}^{13-15}$. Nuestra modalidad de terapia hace prácticamente prescindible la anticoagulación, situación beneficiosa en pacientes críticos.

En nuestro estudio, el $\mathrm{BH}$ promedio fue negativo en $1.006 \mathrm{~mL}$ con una tasa de ultrafiltración de $226 \mathrm{~mL} / \mathrm{h}$ durante un promedio de 4:25 h. Tamme (2015) y Kron (2012) reportaron un BH negativo de $2.900 \mathrm{~mL}$ en $9,4 \mathrm{~h}$, y $4.400 \mathrm{~mL}$ en $10,3 \mathrm{~h}$, es decir, una tasa de ultrafiltración de 309 y de 427 $\mathrm{mL} / \mathrm{h}$ respectivamente ${ }^{14,15}$.

Referente a la tolerancia hemodinámica, reportes en pacientes sépticos muestran disminución de requerimientos de NAD con HDF de alto volumen $^{14,15}$. El $50 \%$ de nuestros pacientes que disminuyeron dosis de NAD cursaban un cuadro séptico. Las elevadas dosis de sustitución de la HDF podrían contribuir a eliminar mediadores inflamatorios, y por ende, favorecer la estabilidad hemodinámica ${ }^{14,15,17,18}$.

Si bien, no incluimos pacientes extremadamente graves, había pacientes críticos con compromiso hemodinámico importante. El aumento de requerimiento de NAD, disminución de PAM o incremento de FC fue marginal; demostrando la excelente tolerabilidad hemodinámica de la HDF intermitente y prolongada en pacientes críticos. Esta idea queda respaldada por la ausencia de diferencia estadística entre los grupos de terapias con distintas dosis de NAD inicial y requerimientos de NAD durante ésta.

La efectividad depurativa es un "outcome" exigible para TRR en paciente crónico, no en enfermos agudos; de hecho, no se asocia con mejores resultados $^{19}$. La dosis depurativa recomendada ${ }^{20} \mathrm{en}$ paciente crítico agudo es un $\mathrm{Kt} / \mathrm{V}$ semanal $>3,9$; que puede ser superado ampliamente con HDF intermitente/prolongada diaria.

La HDF en línea intermitente o prolongada con flujos de alto volumen podría ser una TRR ideal para pacientes críticos, permite mayor depuración cuidando la hemodinamia y sin los riesgos de la anticoagulación sistémica.

El principal aporte de esta investigación es entregar información sobre la HDF en línea intermitente o prolongada en paciente crítico como una modalidad razonable de TRR, considerando los beneficios que entrega como tolerabilidad hemodinámica en pacientes bien seleccionados, disminución de $\operatorname{costos}^{10,12} \mathrm{y}$ menos complicaciones $^{10,14,15}$.

Agradecimientos: A directivos, enfermeras y personal de la Unidad de procedimiento nefrológico participante en este estudio.

\section{Referencias}

1. Singh AT, Mc Causland FR. Osmolality and blood pressure stability during hemodialysis. Semin Dial 2017; 30 (6): 509-17.

2. Daugirdas JT. Dialysis hypotension: a hemodynamic analysis. Kidney Int 1991; 39 (2): 233-46.

3. Gatward JJ, Gibbon GJ, Wrathall G, Padkin A. Renal replacement therapy for acute renal failure. A survey of practice in adult intensive care units in the United Kingdom. Anaesthesia 2008; 63: 959-66.

4. Langford S, Slivar S, Tucker SM, Bourbonnais FF. Exploring CRRT practices in ICU: a survey of Canadian hospitals. Dynamics 2008; 19: 18-23.

5. RENAL Study Investigators. Renal replacement therapy for acute kidney injury in Australian and New Zealand intensive care units: a practice survey. Crit Care Resusc 2008; 10: 225-30.

6. Uchino S, Bellomo R, Morimatsu H, Morgera S, Schetz $\mathrm{M}$, Tan I, et al. Continuous renal replacement therapy: a worldwide practice survey. The beginning and ending supportive therapy for the kidney (B.E.S.T. kidney) investigators. Intensive Care Med 2007; 33: 1563-70.

7. Kellum JA, Angus DC, Johnson JP, Leblanc M, Griffin $\mathrm{M}$, Ramakrishnan N, et al. Continuous versus intermittent renal replacement therapy: a meta-analysis. Intensive Care Med 2002; 28(1): 29-37.

8. Rabindranath K, Adams J, Macleod AM, Muirhead N. Intermittent versus continuous renal replacement therapy for acute renal failure in adults. Cochrane Database Syst Rev 2007; (3): CD003773.

9. Lins RL, Elseviers MM, Van der Niepen P, Hoste E, Malbrain ML, Damas $\mathrm{P}$, et al. Intermittent versus continuous renal replacement therapy for acute kidney injury patients admitted to the intensive care unit: results of a randomized clinical trial. Nephrol Dial Transplant 2009; 24 (2): 512-8.

10. Maduel F, López J. Hemodiafiltración en línea. En: Lorenzo V, López-Gómez JM, Editores, Nefrología al día. España: Editorial Euromedice; 2010. p. 437-45.

11. Van der Sande FM, Kooman JP, Konings CJ, Leunissen KM. Thermal effects and blood pressure response du- 
ring postdilution hemodiafiltration and hemodialysis: the effect of amount of replacement fluid and dialysate temperature. J Am Soc Nephrol 2001; 12 (9): 1916-20.

12. Pérez-García R. ¿Cómo debe ser la hemodiafiltración en línea después del estudio ESHOL? Nefrología 2014; 34 (2): 139-44.

13. AlEnezi F, Alhazzani W, Ma J, Alanazi S, Salib M, Attia $\mathrm{M}$, et al. Continuous venovenous hemofiltration versus continuous venovenous hemodiafiltration in critically ill patients: a retrospective cohort study from a Canadian tertiary centre. Can Resp J 2014; 21: 176-80.

14. Tamme K, Maddison L, Kruusat R, Ehrlich HE, Viirelaid $\mathrm{M}$, Kern $\mathrm{H}$, et al. Effects of high volume haemodiafiltration on inflammatory response profile and microcirculation in patients with septic shock. Biomed Res Int 2015; 2015: 125615.

15. Kron J, Kron S, Wenkel R, Schuhmacher HU, Thieme U, Leimbach T, et al. Extended daily on-line high-volume haemodiafiltration in septic multiple organ failure: a well-tolerated and feasible procedure. Nephrol Dial Transplant 2012; 27: 146-52.

16. Ledebo I, Blankestijn PJ. Haemodiafiltration-optimal efficiency and safety. NDT Plus 2010; 3(1): 8-16.

17. Ronco C, Ricci Z, De Backer D, Kellum JA, Taccone FS, Joannidis $\mathrm{M}$, et al. Renal replacement therapy in acute kidney injury: controversy and consensus. Crit Care 2015; 19 (1): 146.

18. Joannes-Boyau O, Honore PM, Perez P, Bagshaw SM, Grand H, Canivet JL, et al. High-volume versus standard-volume haemofiltration for septic shock patients with acute kidney injury (IVOIRE study): a multicentre randomized controlled trial. Intensive Care Med 2013; 39: 1535-46.

19. Vijayan A, Palevsky P. Dosing of Renal Replacement Therapy in Acute Kidney Injury. Am J Kidney Dis 2012; 59(4): 569-76.

20. Khwaja A. KDIGO clinical practice guidelines for acute kidney injury. Nephron Clin Pract 2012; 120(4): 179-84. 